\title{
Childhood tuberculosis: progress requires an advocacy strategy now
}

\author{
Andreas Sandgren, Luis E. Cuevas, Masoud Dara, Robert P. Gie, \\ Malgorzata Grzemska, Anthony Hawkridge, Anneke C. Hesseling, Beate Kampmann, \\ Christian Lienhardt, Davide Manissero, Claire Wingfield and Stephen M. Graham
}

\begin{abstract}
Childhood tuberculosis (TB) is a preventable and curable infectious disease that remains overlooked by public health authorities, health policy makers and TB control programmes. Childhood TB contributes significantly to the burden of disease and represents the failure to control transmission in the community. Furthermore, the pool of infected children constitutes a reservoir of infection for the future burden of TB. It is time to prioritise childhood TB, advocate for addressing the challenges and grasp the opportunities in its prevention and control. Herein, we propose a scientifically informed advocacy agenda developed at the International Childhood TB meeting held in Stockholm, Sweden, from March 17 to 18, 2011, which calls for a renewed effort to improve the situation for children affected by Mycobacterium tuberculosis exposure, infection or disease. The challenges and needs in childhood TB are universal and apply to all settings and must be addressed more effectively by all stakeholders.
\end{abstract}

KEYWORDS: Control, infectious disease, Mycobacterium tuberculosis, public health $t$ is estimated that of the nine million tuberculosis (TB) cases occurring every year worldwide, one million occur among children [1]. However, estimates of the burden of childhood TB are uncertain, and under-reporting and underestimation of the global numbers are likely. In most settings, children with TB continue to be given low priority by National TB control Programmes (NTP) because they are less likely to transmit disease. Provision of more accurate data by national surveillance programmes is essential for improved global TB-burden estimates among children. Childhood TB has been largely absent from the global public health agenda despite being a major contributor to childhood morbidity and mortality particularly in high TB-burden settings and advocacy and scientific progress are still insufficient. Guidelines for childhood TB management have been developed and are already adopted by many countries. However, a wide policy/practice gap remains that needs to be addressed. Advocacy for the implementation and monitoring of these policies is, therefore, critical.

\section{THE INTERNATIONAL CHILDHOOD TB MEETING}

The first International Childhood TB meeting dedicated to advocacy was organised by the Stop
TB Partnership and the European Centre for Disease Prevention and Control and was held in Stockholm, Sweden, from March 17 to 18, 2011. The meeting highlighted the challenges and successes in research and control of childhood TB and identified key areas where advocacy and targeted engagement with stakeholders is needed. The aim was to identify the advocacy priorities for childhood TB and to bring forward the voice of the children. The meeting was attended by over 110 participants representing the global TB control community, paediatricians, researchers, former patients, civil society, global health activists, advocacy experts, donors, governmental agencies and industry. This broad audience engaged in discussions and shared views on how best to raise the awareness of childhood TB.

\section{CHALLENGES AND NEEDS IN RESEARCH AND DEVELOPMENT FOR CHILDHOOD TB}

Research needs to address the unique aspects of childhood TB and needs to function as an essential vehicle towards the elimination of TB. Children are more susceptible than adults to progress from Mycobacterium tuberculosis exposure to infection and to disease, which implies that the immune mechanisms mediating protection might be different compared to those in adults. To increase our
AFFILIATIONS

For author affiliations, please refer to the Acknowledgements section.

CORRESPONDENCE

A. Sandgren

European Centre for Disease

Prevention and Control

Tomtebodavagen 11a

Stockholm

17183

Sweden

E-mail: andreas.sandgren@

ecdc.europa.eu

Received:

Oct 272011

Accepted after revision:

Dec 132011

First published online:

Feb 152012

This article was modified in April 2016 to correct errors in the licence information. 
understanding of the underlying immune phenomena requires the identification of markers of protection or susceptibility and the development of laboratory methods and diagnostics applicable to children [2]. The current lack of suitable diagnostic reference standards is, however, a barrier for involving children in research. Deficiencies in suitable diagnostics equally represent a challenge for the rapid detection and care of children with TB. New diagnostic methods, including the more sensitive direct fluorescence sputum microscopy techniques, faster liquid culture, rapid speciation methods and automated nucleic acid amplification tests are currently insufficiently evaluated or may not be suitable for young children, as they rarely produce sputum and often have paucibacillary disease.

Children were historically excluded from TB drug trials due to perceived difficulties in case definitions and markers of treatment response. Their treatment is therefore primarily based on the adaptation of drug regimens designed for adults. While there are new World Health Organization (WHO) recommendations for improved childhood TB treatment regimens [3], there is a lack of child-friendly formulations matching the new WHO recommendations. Furthermore, the identification and treatment of children with drug-resistant TB is often based on limited clinical experience and scarce scientific evidence. The inclusion of children early in the drug development pathway, with prioritisation of assessment of their pharmacokinetics, safety and drug formulation, is critical for the development of child-friendly and safe treatment regimens.

The Bacille Calmette-Guérin (BCG) vaccine protects young children against severe forms of the disease (e.g. TB meningitis) and disseminated TB $[4,5]$. However, BCG has variable efficacy against pulmonary $\mathrm{TB}$ and severe adverse events in HIVinfected infants have been reported [6]. BCG vaccination is therefore recommended to be withheld from infants with known HIV-positive status while in infants born to HIV-infected mothers and unknown status, the risks and benefits need to be considered [7]. Although, the benefits of BCG in most settings and populations clearly outweigh its risks [7], there is an urgent need for a more effective and safer TB vaccine for children [8]. Vaccine development would be greatly facilitated by the identification of immune correlates of protection, the availability of accurate diagnostic tests for the definition of suitable endpoints for efficacy and the development of additional paediatric vaccine trial sites.

\section{CHALLENGES AND NEEDS IN PROGRAMMATIC TB CONTROL FOR CHILDHOOD TB}

The basis for establishment of effective childhood TB control is available and is supported by evidence and best practices. This includes active case-finding of children in contact with adults with TB, BCG vaccination, diagnosis of TB, isoniazid preventive therapy to prevent progression of latent TB and treatment of active disease. Guidelines that focus on the management of TB in children have been developed in the last $5 \mathrm{yrs}$, and provide a framework for NTPs [9]. Despite the fact that these elements provide the basis for the development of national, regional and global comprehensive childhood TB control, broader and systematic implementation has not yet occurred. Thus, activities to address childhood TB remain fragmented and are often outside the scope of national and regional strategies. This creates a policy/practice gap in addressing control and prevention of TB among children. There is therefore a need to enhance adoption of international guidelines and to implement and scale these up within NTPs.

\section{ADVOCACY STRATEGIES IN CHILDHOOD TB The need for advocacy}

The activist movement, which has been key to driving the global response to HIV / AIDS for over 25 yrs, was non-existent in TB control until recently [10]. This is particularly true for childhood TB, which is an indication of failing TB control in the community [11]. However, childhood TB is often neglected by scientists, donors, policy makers, healthcare professionals, product developers and advocates. This is in sharp contrast with the sense of urgency and innovation that has marked the response to paediatric HIV. For example, most therapies approved to treat HIV have paediatric dosing ranges and child-friendly formulations [12] while there is a dearth of evidence for the guidelines on TB treatment for children [13] and a lack of tools to ease treatment of TB in children. Thus, this vulnerable and neglected population require constant and effective advocacy on its behalf to address some of the major challenges (table 1).

\section{Advocacy messages}

TB in children is usually not microbiologically confirmed for a number of well-documented reasons. While concepts frequently used such as "smear negative" or "paucibacillary" may not be difficult to understand for the medical professionals, they do require explanation as these concepts can complicate simple messages to the broader community. Childhood TB advocates need to use language that is accessible and appeals to a broad audience. Furthermore, messaging should shift from what is lacking to what is needed and what is possible. While there is undoubtedly a need for improved diagnostic tools and therapeutic evidence, especially with second-line drugs, it is important to recognise that if a diagnosis can be made and a consistent supply of properly dosed medication is available, most cases of childhood TB can be cured using current drugs [14]. Advocacy messages should highlight effective interventions and demand their implementation, and bring focus to gaps in care and the need for newer and better tools to prevent, diagnose and treat TB in children of all ages (table 2).

TABLE 1 Major challenges of childhood tuberculosis (TB)

TB is an important cause of child morbidity and mortality, the low priority awarded to childhood TB due to its lower infectivity is inexcusable

Accurate surveillance data regarding the burden of TB in children are scarce because it is under-detected and under-reported, this has implications for the quality of patient care and the recognition and attention awarded to childhood TB

There is a lack in research and development of improved diagnostic tools, new drugs, easy-to-dose formulations and effective vaccines to prevent all forms of TB in children of all ages

Donor interest is lacking and research and programmatic control are under funded 


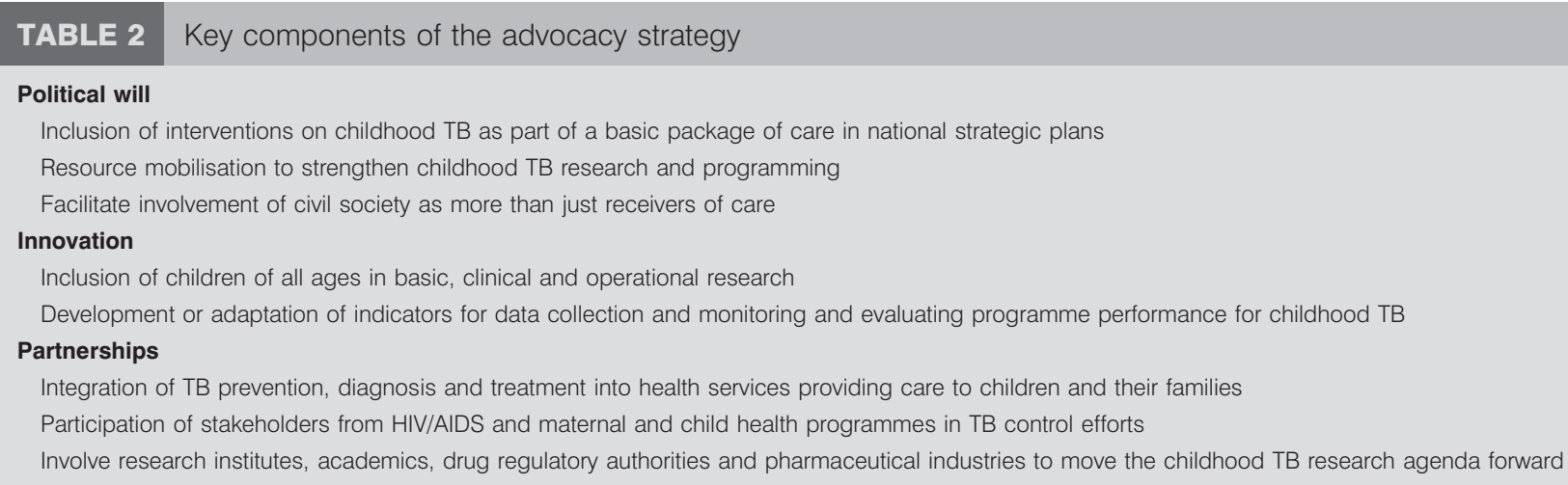

TB: tuberculosis.

\section{Partnerships}

TB transmits readily within the household and infants and young children are at high risk to acquire the infection from their family members. Specifically, children whose mothers are co-infected with HIV have an increased risk of TB [15, 16]. Given the family-based nature of the disease, TB services such as case-finding, preventive therapy and treatment require better interaction between TB programmes and HIV/AIDS, immunisation, and child and maternal health programmes. Thus, TB advocates must collaborate with colleagues from these other fields to ensure that childhood TB is an integral part of their practice and policy.

\section{Monitoring and evaluation}

$\mathrm{TB}$ is a disease of poverty and yet there is no specific mention of childhood or maternal TB in the Millennium Development Goals (MDGs), including MDG 4 (reduce child mortality) and MDG 5 (improve maternal health). Although there are two TB indicators included in MDG 6 (combat HIV/AIDS, malaria and other diseases), there is no reference to tracking childhood or maternal TB.

The US President's Emergency Plan for AIDS Relief has included indicators of prevention, diagnosis and treatment of childhood and maternal TB as essential programme components in its guidance to countries [12]. These should be adopted across global institutions such as the WHO, UN agencies, and the Global Fund to Fight AIDS, Tuberculosis and Malaria to establish a uniform framework for monitoring and evaluating the implementation of childhood and maternal TB interventions.

\section{A CALL TO ACTION}

Childhood TB will remain neglected without strong activism. Advocates for childhood TB representing a broad spectrum of public health, research, donors and civil society, and equipped with the most robust, relevant and up-to-date epidemiological, clinical and basic science data, must act with a sense of urgency, outrage and hopefulness. With this in mind, participants at the International Childhood TB meeting in Stockholm issued a "Call to Action for Childhood TB" [17], the Call to Action can still be signed via the Stop TB Partnership website [18]. It has so far been endorsed by over 800 individuals and organisations from nearly 100 countries. The call urges the international community to commit to addressing the needs of children at risk for and suffering from TB.

\section{SUPPORT STATEMENT}

L.E. Cuevas, M. Grzemska and C. Lienhardt are staff of the World Health Organization. The authors of this paper alone are responsible for the views expressed in this publication which do not necessarily represent the decisions or policies of the World Health Organization. R.P. Gie, A. Hawkridge, A.C. Hesseling, B. Kampmann, C. Lienhardt, C. Wingfield and S.M. Graham received support from the European Centre for Disease Prevention and Control (Stockholm, Sweden) or the Childhood TB subgroup, Stop TB Partnership (Geneva, Switzerland) for travel and accommodation to attend the International Childhood TB meeting.

\section{STATEMENT OF INTEREST}

A statement of interest for A. Hawkridge can be found at www.erj. ersjournals.com/site/misc/statements.xhtml

\section{ACKNOWLEDGEMENTS}

Author affiliations are as follows: A. Sandgren and D. Manissero, European Centre for Disease Prevention and Control, Stockholm, Sweden; L.E. Cuevas, UNICEF/UNDP/World Bank/WHO Special Programme for Research and Training in Tropical Diseases, Geneva, Switzerland; M. Dara, World Health Organization, Regional Office for Europe, Copenhagen, Denmark; R.P. Gie and A.C. Hesseling, Desmond Tutu TB Centre, Dept of Paediatrics and Child Health, Faculty of Health Sciences, Stellenbosch University, Stellenbosch, South Africa; M. Grzemska and C. Lienhardt, Stop TB Partnership, Stop TB Department, World Health Organization, Geneva, Switzerland; A. Hawkridge, Institute of Infectious Disease and Molecular Medicine, University of Cape Town, Cape Town, South Africa; B. Kampmann, Dept of Paediatrics, Imperial College, London, UK, and Medical Research Council Unit The Gambia, Banjul, Gambia; C. Wingfield, Treatment Action Group, New York, NY, USA; S.M. Graham, Centre for International Child Health, University of Melbourne, Dept of Paediatrics and Murdoch Children's Research Institute, Royal Children's Hospital, Melbourne, Australia, and International Union Against Tuberculosis and Lung Disease, Paris, France.

\section{REFERENCES}

1 Nelson LJ, Wells CD. Global epidemiology of childhood tuberculosis. Int J Tuberc Lung Dis 2004; 8: 636-647.

2 Jones C, Whittaker E, Bamford A, et al. Immunology and pathogenesis of childhood TB. Paediatr Respir Rev 2011; 12: 3-8. 
3 World Health Organization. Rapid advice: treatment of tuberculosis in children. WHO/HTM/TB/2010.13. Geneva, WHO, 2010.

4 World Health Organization. BCG vaccine. WHO position paper. Wkly Epidemiol Rec 2004; 79: 27-38.

5 Trunz BB, Fine P, Dye C. Effect of BCG vaccination on childhood tuberculous meningitis and miliary tuberculosis worldwide: a meta-analysis and assessment of cost-effectiveness. Lancet 2006; 367: 1173-1180.

6 Hesseling AC, Johnson LF, Jaspan H, et al. Disseminated bacille Calmette-Guérin disease in HIV-infected South African infants. Bull World Health Organ 2009; 87: 505-511.

7 [Revised BCG vaccination guidelines for infants at risk for HIV infection]. Wkly Epidemiol Rec 2007; 82: 193-196.

8 Kaufmann SH. Future vaccination strategies against tuberculosis: thinking outside the box. Immunity 2010; 33: 567-577.

9 World Health Organization. Guidance for national tuberculosis programmes on the management of tuberculosis in children. WHO/HTM/TB/2006.371. Geneva, WHO, 2006.

10 Harrington M. From HIV to tuberculosis and back again: a tale of activism in 2 pandemics. Clin Infect Dis 2010; 50: Suppl. 3, S260-S266.

11 Swaminathan S, Rekha B. Pediatric tuberculosis: global overview and challenges. Clin Infect Dis 2010; 50: Suppl. 3, S184-S194.
12 The United States President's Emergency Plan for AIDS Relief. PEPFAR Guidance on Integrating Prevention of Mother to Child Transmission of HIV, Maternal, Neonatal, and Child Health and Pediatric HIV Services. January, 2011. www.pepfar.gov/guidance/ pmtct/158785.htm Date last accessed: September 16, 2011.

13 Burman WJ, Cotton MF, Gibb DM, et al. Ensuring the involvement of children in the evaluation of new tuberculosis treatment regimens. PLoS Med 2008; 5: e176.

14 Frydenberg AR, Graham SM. Toxicity of first-line drugs for treatment of tuberculosis in children: review. Trop Med Int Health 2009; 14: 1329-1337.

15 Gupta A, Bhosale R, Kinikar A, et al. Maternal tuberculosis: a risk factor for mother-to-child transmission of human immunodeficiency virus. J Infect Dis 2011; 203: 358-363.

16 Gupta A, Nayak U, Ram M, et al. Postpartum tuberculosis incidence and mortality among HIV-infected women and their infants in Pune, India, 2002-2005. Clin Infect Dis 2007; 45: 241-249.

17 Stop TB Partnership. Call to Action for Childhood TB. www. stoptb.org/getinvolved/ctb_cta.asp Date last accessed: September 16, 2011.

18 Stop TB Partnership. Call to Action for Childhood TB. Join the call. www.stoptb.org/getinvolved/cta_signup.asp Date last accessed: September 16, 2011. 